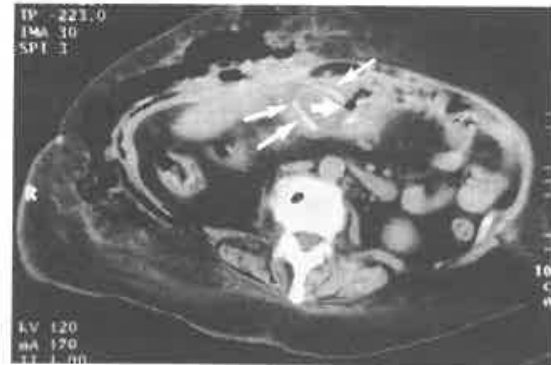

Abb. 2 CT: massive, gasgefüllte subkutane Abszeßstraßen im Oberbauch rechts, Ausläufer zur autochthonen Rückenmuskulatur. Teils kaum von der Bauchwand und angrenzenden Darmschlingen abgrenzbare, partiell wandverkalkte Gallenblase mit solidem Inhalt (lange Pfeile); undeutliche Erkennbarkeit der cholezystoduodenalen Fistel (kurzer Pfeil).

(koinzidentes) Gallenblasenkarzinom nicht auszuschließen.

\section{OP-Befund, Postop. Verlauf, Obduktionsbericht (Auszüge)}

Inzision der Bauchwand paramedian rechts: spontane Entleerung von über zwei Liter Eiter, mit Ausdehnung von Abszeßtaschen bis zur Wirbelsäule. Nekrotisch aufgelöster Musculus rectus abdominis, mit direkt in die Gallenblase mündendem Zugang ins Abdomen. OPSitus: entzündlicher Konglomerattumor im rechten Oberbauch, Bild einer eitrigen Cholezystitis mit gedeckter Perforation in Musculus rectus und Subkutis sowie in Duodenum und angrenzenden, drei große Konkremente enthaltenden Dünndarm, bei beginnendem Gallenstein-Ileus. Absetzen dieses Dünndarmabschnittes außerhalb der entzündlichen Veränderungen, End-zu-EndAnastomose. Übernähung des Duodenaldefektes. Sackförmig erweiterter Ductus hepatocholedochus, völlig aufgebrauchter Ductus cysticus. Intraoperative Cholangiografie: Infundibulum-naher Gallenblasenanteil nicht von einer Ductus Choledochus-Struktur unterscheidbar, daher Absetzung der Gallenblase am Gallenblasenhals, dort Übernähung und retrograde Cholezystektomie. Resektion der nekrotischen Anteile des Musculus rectus. Postoperativ unbeherrschbare generalisierte Staphylokokken-Sepsis, trotz Dauerspülung der breit offenen und mehrfach gegeninzidierten Abszeßhöhle. Exitus der Patientin am 11. postoperativen Tag. Autopsie: ausgedehnte Bauchdecken-Nekrosen und -Phlegmone, Staphylokekkensepsis und Pulmonalembolie.

\section{Diskussion}

Der Hinweis, daß die Ursache für ein spontanes thorakales Weichteilemphysem auch „von unten“ kommen kann, drängt sich für den den Thoraxübersichts-Film befundenden Radiologen zwar nicht sofort auf; je vergeblicher jedoch die Suche nach intrathorakalen Quellen und je „abdomineller" die Klinik ist, desto stärker erhärten sich - zusammen mit der in Richtung Abdomen hinunterwandernden Empyhsemstraße - die Indizien einer abdominellen, in unserem Falle cholezystogenen Komplikation als Ursache für den Thoraxröntgen-Befund.

Zu den häufigsten Komplikationen der akuten Cholezystitis zählen die Konversion in die empyematöse und gangränöse Form, die biliäre Sepsis sowie die freie und die - etwa ins Leberbett oder angrenzende Bauchwand - gedeckte Perforation bzw. die enterogen einbrechende Perforation mit spontaner biliodigestiver Fistel - in Einzelfällen zum Kolon (Chepcheruk et al., Vestn Khir Im II Grek 1992;148:62) - sowie (im Falle meist koinzidenter Cholezystolithiasis) ein Gallensteinileus.

Weniger übliche Kompliktionen der akuten Cholezystitis sind perihepatale Ab- szesse (Forbes et al., Am J Gastroenterol 1996;91:786), retroperitoneale Abszesse (Shimanuki et al., Fikushima J Med Sci 1997;43:113), oder Pfortaderthrombosen (Kidney et al., Clin Radiol 1998,53: 459).

Ein - entsprechend unserer Kasuistik riesiges spontanes abdomino-thorakales Weichteilemphysem als Folge einer in die Bauchwand perforierten gangränösen Cholezystitis stellt einen besonders ungewöhnlichen Befund dar. Differentialdiagnostisch ist beim spontanen abdomino-thorakalen Weichteilemphysem im Falle fehlender intraabdomineller bzw. intrathorakaler Infektionsherde bzw. intrathorakaler pathologischer Luftansammlungen wohl in erster Linie an subkutane Infektionen bzw. Phlegmonen mit gasbildenden Erregern (v. a. Diabetiker) zu denken.

Der Lehrwert des Falles liegt darin, bei spontanen thorako-abdominellen Weichteilemphysemen ohne offensichtliche intrathorakale Quelle auch eine abdominelle Komplikation bzw. bei rechtsseitiger Emphysemlokalisation auch eine cholezystogene Komplikation in Betracht zu ziehen.

I. Gruber, M. Hermann, M. Schratter, Wien

\title{
Pseudoaneurysma der A. epigastrica inferior nach laparoskopischer Cholezystektomie
}

Das Pseudoaneurysma (Aneurysma spurium) ist eine bekannte Folge traumatisch bedingter Gefäßwandschäden. Ursächlich kommen gefäßchirurgische Eingriffe, arterielle Punktionen und nichtiatrogene, meist pfählende Verletzungen in Frage. Dabei gelangt Blut über ein Leck in der Gefäßwand in den extravasalen Raum und wird dort von einer bindegewebigen Kapsel begrenzt. Pseudoaneurysmen der A.epigastrica inferior sind selten und im Zusammenhang mit einer laparoskopischen Cholezystektomie in der Literatur bisher nicht beschrieben. Wir berichten über den Fall eines Aneurysma spurium der A. epigastrica inferior dextra nach laparoskopischer Cholezystektomie und diskutieren Pathogenese, Diagnostik und Therapie.

\section{Fallbeschreibung}

Ein 61jähriger Patient wird mit unklaren abdominellen Beschwerden bei Zustand nach laparoskopischer Cholezystektomie eingewiesen. Die Operation erfolgte vor 10 Wochen wegen einer symptomatischen Cholezystitis bei Cholezystolithiasis. Unmittelbar postoperativ trat damals eine transfusionspflichtige Nachblutung aus dem Gallenblasenbett auf, welche Anschluß an eine hier liegende Drainage hatte und konservativ beherrscht wurde. Am 6. postoperativen Tag mußte ein infiziertes Hämatom im Bereich der Nabelinzision eröffnet werden, welches im weiteren Verlauf komplikationslos sekundär ausheilte.

Es bestanden folgende Vorerkrankungen: Terminale, dialysepflichtige Nieren-

insu riph

tain

Refl

herı

sow

Bei

Bau

Übe

erg

ger

recl

Res

bag

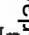

.

H更

bi 
insuffizienz infolge Nephrosklerose, periphere AVK bds. Stadium III nach Fontaine, hämorrhagische Refluxösophagitis III bei axialer Hiatushernie, renale Anämie und Osteopathie sowie Zustand nach Lungentuberkulose.

Bei Aufnahme klagte der Patient über Bauchschmerzen und rezidivierende Übelkeit. Die klinische Untersuchung ergab weiche Bauchdecken und einen geringen Druckschmerz paraumbilical rechts. Hier war eine ca: $5 \mathrm{~cm}$ große Resistenz rechts lateral des Nabels tastbar.

In der Röntgen-Abdomenübersichtsaufnahme a.p. im Stehen fanden sich keine Hinweise auf freie Luft, keine Spiegelbildung, keine stehenden Darmschlingen. Es bestand ein massiv stuhlgefüllter Kolonrahmen als Hinweis auf eine Koprostase.

Unter dem Verdacht auf ein Bauchwandhämatom oder eine Abszeßbildung wurde eine abdominelle Sonographie durchgeführt. Hier zeigte sich in topographisch-anatomischer Korrelation zum Tastbefund eine glatt begrenzte, leicht inhomogene und überwiegend echoarme Raumforderung in der vorderen Bauchwand mit einer Größe von $6 \times 5 \times 5 \mathrm{~cm}$, welche im Farb-DopplerBild partiell arterielle Flußsignale aufwies (Abb.1).

Computertomographisch bestätigte sich der Befund als kugelige, kapselartig begrenzte Raumforderung in der vorderen Bauchwand extraperitoneal mit einer Größe von $6 \times 5 \times 4,5 \mathrm{~cm}$ (Abb. 2). Die dorsalen Anteile zeigten eine homogene hypodense Binnenstruktur, ventral war nach intravenöser Kontrastmittelapplikation ( $120 \mathrm{ml}$ Imeron 300, Fa. Byk Gulden, Konstanz) ein deutliches Enhancement auf Werte über 120 HounsfieldEinheiten abzugrenzen. Es zeigte sich eine Anhebung und Kompression des Musculus rectus abdominis, angrenzende Dünndarmschlingen waren ebenfalls verlagert. Miterfaßt wurden beiderseits zystisch degenerierte Nieren. Anhand der vorliegenden Befunde wurde die Diagnose eines teilthrombosierten Pseudoaneurysmas der Arteria epigastrica inferior gestellt.

Aufgrund der Größe des Pseudoaneurysmas mit der daraus resultierenden abdominellen Symptomatik und wegen der bestehenden terminalen Niereninsuffi-

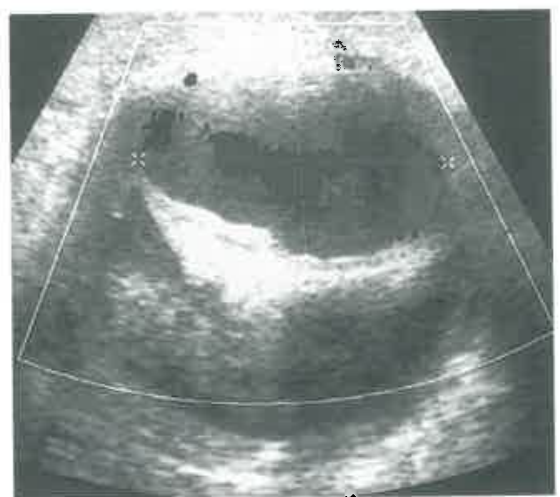

Abb.1 Farbkodierte Doppler-Sonographie, paraumbilikaler Längsschnitt; überwiegend glatt begrenzte echoarme Raumforderung mit zentralem arteriellen Flußsignal.

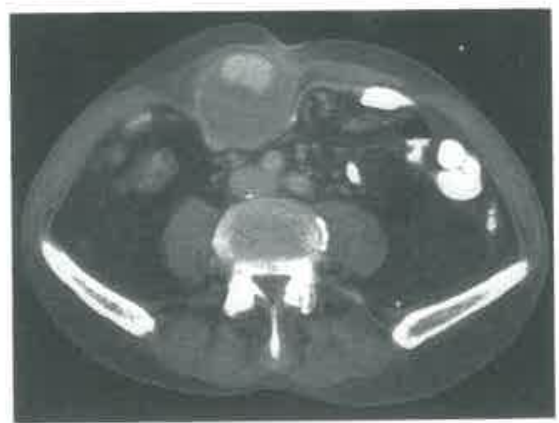

Abb. 2 Kontrastverstärkte abdominelle Spiral-CT nach oraler Darmkontrastierung; teilthrombosiertes Pseudoaneurysma an der vorderen Bauchwand mit ventral gelegenen perfundierten Anteilen.

zienz erfolgte eine chirurgische Exstirpation mit vorheriger Umstechung der A. epigastrica inferior oberhalb und unterhalb des Aneurysmas.

\section{Diskussion}

Das Pseudoaneurysma ist durch ein "Leck" in der Arterienwand gekennzeichnet, durch das es zu einer Verbindung zwischen perivasalem, teils organisiertem und endothelialisiertem Hämatom und Gefäßlichtung kommt. Für solche Gefäßverletzungen sind in zunehmendem Maße iatrogene Ursachen verantwortlich. Dabei standen früher vorwiegend gefäßchirurgische Eingriffe im Vordergrund, in den letzten Jahren ist ein großer Anteil der publizierten Pseudoaneurysmen auf minimal-invasive perkutane Techniken zurückzuführen. Beleg dafür ist ein deutlicher Anstieg der Anzahl an Veröffentlichungen über Pseudoaneurysmen, dabei handelt es sich überwiegend um Fallberichte. Die steigende Inzidenz an Pseudoaneurysmen steht in direktem Zusammenhang mit den zunehmenden perkutanen diagnostischen und therapeutischen Maßnahmen, an erster Stelle den transfemoralen Katheterangiographien.

Bisher liegen in der Literatur nur wenige Berichte über ein Aneurysma spurium der Arteria epigastrica inferior vor, jeweils Fallbeschreibungen über Komplikationen ärztlicher Eingriffe. Ursachen waren die Entfernung eines TenckhoffKatheters (Werner et al, Nephrol Dial Transplant. 1999;14:1297), Aszitespunktionen (Lam et al, J Vasc Surg. 1998; 28:566) und chirurgische Stütznähte bzw. deren verzögerte Entfernung nach Laparotomie (Ferrer et al, Arch Surg. 1996). Ein Pseudoaneurysma der A. epigastrica inferior nach laparoskopischer Cholezystektomie ist bisher nicht beschrieben. Als Grund für die Entstehung des Pseudoaneurysmas kommt im vorliegenden Fall eine Verletzung des Gefäßes durch Trokarmanipulationen in Betracht, obwohl der Zugang korrekt in der Medioclavicularlinie gewählt wurde. Bei schlanken Patienten können die Bauchdeckengefäße durch eine Translumination der Bauchwand von innen mit der Kameraoptik erkannt und weitere Trokare unter Sicht eingebracht werden, was hier offenbar nicht zur Vermeidung der Gefäßläsion geführt hat.

Die Komplikationsraten nach laparoskopischer Cholezystektomie liegen nach Literaturangaben mit 3-11\% deutlich unter denen nach offener Gallenblasenentfernung (14-42\%), wobei die Häufigkeit und Charakteristik unter anderem von der Schwere der Entzündung und der Erfahrung des Operateurs abhängig ist (Hannan et al, Surgery 1999;125: 223).

Im beschriebenen Fall wurde die Symptomatik des Patienten durch lokale Schmerzen und eine peritoneale Reizung bestimmt. Die initialen Differentialdiagnosen eines Bauchwandhämatomes oder Abszesses waren vorwiegend klinisch und anamnestisch begründet. Mit Farb-Doppler-Sonographie und kontrastverstärkter Spiral-CT konnte die definitive Diagnose einwandfrei gestellt werden. Eine angiographische Darstellung des Pseudoaneurysmas war nicht notwendig, zumal eine interventionellradiologische Therapie (Embolisation) wegen der Größe des Befundes und der terminalen Niereninsuffizienz aus unserer Sicht nicht indiziert war. 
Bei unklaren Bauchwandverdickungen nach Punktionen oder laparoskopischen Eingriffen sollte an das Vorliegen eines Pseudoaneurysmas der A. epigastrica inferior oder superior gedacht werden. Therapeutisch kommt die Katheterembolisation (Glanz et al, Cardiovasc Intervent Radiol 1987;10:198) oder die chirurgische Exstirpation in Frage; die Indikation hierzu sollte im Einzelfall interdisziplinär gestellt werden.
Die Kenntnis der Ursachen und Entstehungsmöglichkeiten eines Pseudoaneurysmas ist auch im Hinblick auf die zunehmende Bedeutung der CT- oder Ultraschall-gesteuerten Punktionen und Drainagen für den Interventionellen Radiologen von Bedeutung.

\section{R. Wutke, T. Reck, W. Bantz, Erlangen}

\section{Nicht parasitär bedingter renaler Lymphreflux mit Chylurie}

Die Lymphurie bzw. Chylurie weist in den Tropen und subtropischen Gebieten eine hohe Inzidenz auf. In Europa stellt sie ein sehr seltenes Krankheitsbild dar häufig als Pyurie oder Lipidurie fehlgedeutet. Vor der Einführung der Lymphographie durch Kinmonth 1952 konnte die Lymphurie häufig nur zystoskopisch nach einer Provokationsmahlzeit nachgewiesen werden; die Urographie zeigte meist einen Normalbefund. Eine weitere diagnostische Möglichkeit stellte die orale Gabe von Sudan III-gefärbtem Fett dar - bei renalem Lymphreflux kam es zu einer Rotfärbung des Urins.

Durch die Lymphographie ist es möglich geworden, die Morphologie des lymphatischen Systems bei der Chylurie in vivo genauer $\mathrm{zu}$ studieren. Sie ermöglicht eine exakte Aussage zur Lokalisation und Ausdehnung der Lymphsystemveränderungen sowie über das Vorliegen eines Refluxes. Im folgenden soll mit der Präsentation eines nicht-parasitären renalen Lymphrefluxes ein ungewöhnlicher Fall, diagnostiziert durch ein seltener gewordenes Untersuchungsverfahren, dargestellt werden.

\section{Kasuistik}

Eine 32jährige Patientin stellte sich aufgrund eines weißlich-trüben Urins mit Schleimabgang vor. Dieses Ereignis sei seit der Geburt ihres zweiten Kindes vor einem Jahr wiederholt aufgetreten; intermittierend hätten rechtsseitige Flankenschmerzen das Geschehen begleitet. Ein ähnliches Beschwerdebild habe auch nach der Geburt ihres ersten Kindes vor 6 Jahren bestanden und nach Antibiotikagabe sistiert.
Anamnestisch fanden sich außer rezidivierenden Urozystitiden keine Besonderheiten. Bei Aufnahme lag kein Fieber vor, der Urin war klar, und die Blutlaborwerte befanden sich im Normbereich. Sonographisch zeigte sich eine Weitstellung des rechten Nierenbeckens, die allerdings nach einigen Tagen nicht mehr nachweisbar war, vereinbar mit einer symptomatischen Harnstauungsniere bei einem Fibrinkoagel im rechten Ureter. Die Erregersuche (u.a. Mykoplasmen, Ureaplasmen, Tbc) verlief negativ.

Zystoureteroskopisch wurde ein schleimartiges Gebilde aus der Blase entfernt, ansonsten war der Befund bis auf eine weißliche Urinfahne aus dem rechten Ostium unauffällig. Die mikrobiologische Untersuchung des gewonnenen Materials konnte keinen Keimnachweis erbringen. Histologisch ließ sich ausschließlich Fibrin darstellen; es lag kein Hinweis auf Malignität vor. Die Computertomographie der Nieren, nativ und nach intravenöser Kontrastmittelapplikation (Doppelspiraltechnik), war unauffällig.

Die chemische Analyse des Urins zeigte einen erhöhten Fett- und Proteingehalt, vereinbar mit einer Lymphurie. Eine Filariose konnte sowohl anamnestisch als auch klinisch ausgeschlossen werden. Lymphographisch (pedale maschinelle Injektion von je $8 \mathrm{ml}$ Lipiodol Ultra-Fluid, Byk-Gulden, Konstanz) zeigte sich ein chylöser Reflux aus dem rechten Truncus lumbalis über die rechtsseitigen, ektatischen renalen Lymphgefäße in das Nierenhohlsystem (Abb.1 a, b). Es lag allenfalls eine geringe Seitendifferenz in der Ausprägung der retroperitonealen Lymphknoten mit geringfügig schwä-
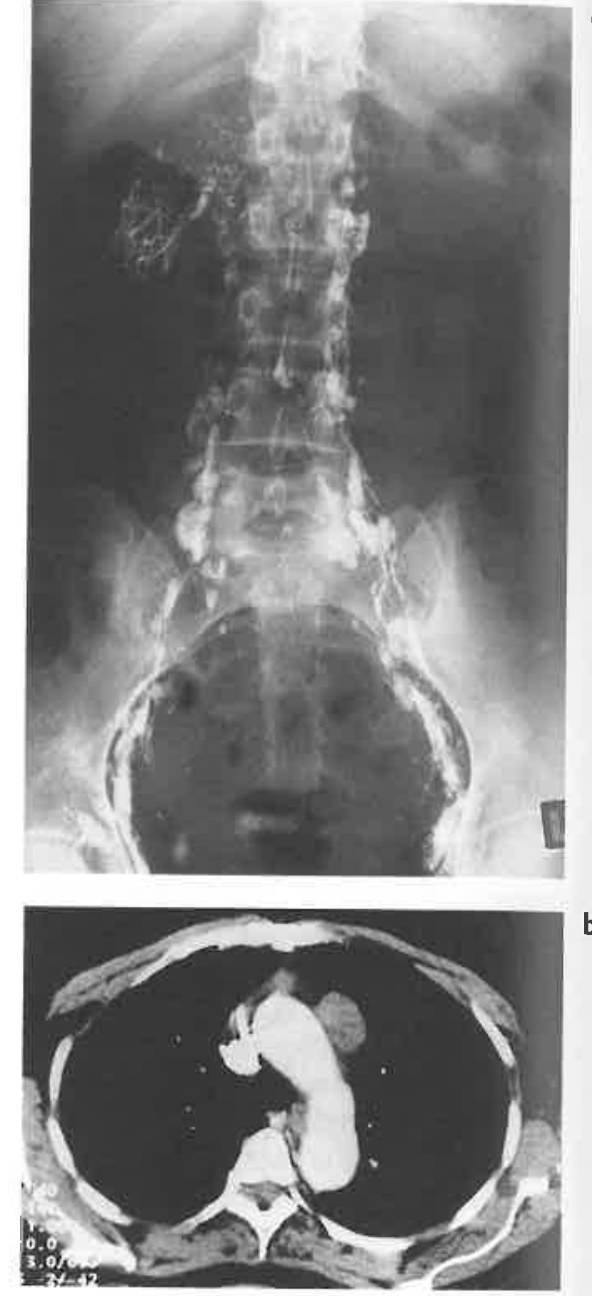

Abb.1 (a) Lymphographische Darstellung der Lymphknoten und -bahnen im Bereich des Beckens und paravertebral. Etwas verminderte Kontrastierung der paralumbalen Lymphknoten re. sowie ein deutlich ausgeprägter Kontrastmittelreflux über ektatische renale Lymphgefäße re. (b) Vergrößerung aus a) mit Detaildarstellung der ektatischen renalen Lymphgefäße rechtsseitig.

cherer Kontrastierung rechts vor. Es ergab sich kein Hinweis auf einen tumorösen Befall des retroperitonealen Gewebes oder auf entzündlich bedingte LK-Veränderungen. Auch Zeichen der Obstruktion wie Kollateralkreisläufe konnten nicht dargestellt werden, der Ductus thoracicus war regelrecht abzugrenzen.

Intraoperativ ließen sich mehrere kräftige Lymphbahnen im Sinne von Fisteln darstellen, die anschließend ligiert wurden. Weiterhin zeigte sich eine auffällige Blutversorgung mit vielen fächerförmigen kleinen Nierenarterien ohne arteri- 